\title{
A PROBLEM EROM THE THEORY OF OBSERVABILITY
}

\author{
M.S.NIKOLSKII \\ Steklov Institute of Mathematics, \\ Academy of Sciences of the USSR, Moscow
}

When studying control problems, the information aspect is important. What do we know about the motion and what is it possible to know from the available information for the purpose of control?

In classical models of the optimal control theory and of the theory of differential games, the presence of a large amount of information about the motion and the dynamics of objects is postulated. In practice, such information is not always accessible because of technical reasons. Perhaps it is for this reason that the theory of observability and of filtration arose inside the control theory.

One of the first problems studied in the theory of observability was Kalman's problems about observation of a linear object. The problem is discussed below. Later N.N.Krasovskil studied the problem of the best observability of a linear system with disturbances in measurement. Many papers were published in recent years on problems of the optimal control theory and the theory of differential games with incomplete information. As a rule, these works did not do specially with the observable aspect, but the latter was taken into account under the exact formalization of process under study. We will mention here the works of N.N.Krasovskii, A.B.Kuržanskii, Yu.S.Osipov, F.L.Chernous'ko, A.A. Melikjan, I.Ya.Kač, V.B.Kolmanovskii, G.S.Selementjev and other authors. The problem of combination of observation and control was studied specially in some of these papers (see, for example, the works of A.B.Kuržanskil, A.A.Melikjan, V.B.Kolmanovskii, G.S.Selementjer and others). 
The aim of this paper is to study the problem of observability arising in pursuit differential games in the case when the phase vector of the evading object is not known exactly. I have begun studying such games on the advice of I.S.Pondrjagin.

\section{Kalman's problem of observability}

In the $n$-dimensional Fuclidean space $R^{n}$, motion of the Iinear object takes place in accordance with the differential equation

$$
\dot{x}=A x+B u \text {, }
$$

where $x \in R^{n}, x(0)=x_{0}, u \in R^{P} ; A, B$ are constant matrices; $u=u(t)$ is an arbitrary measurable Lebegue's summable function.

The following signal is accessible for observer's measurement

$$
y(t)=G \times(t) \quad, \quad 0 \leq t \leq \Delta,
$$

where $y \in R^{m}, G$ is the constant matrix, $\Delta>0$. It is assumed that the observer knows $A, B, G$ and $y(t), u(t)$ on $0 \leq t \leq \Delta$. His aim is to find $x(0)=x_{0}$.

\section{The Problem of ideal observability.}

It is assumed here unlike the Kalman's problem that the observer does not know $u(t)$ on $0 \leq t \leq \Delta$. His aim is to find $x(0)=x_{0}$.

The class of ideal observable systems belongs to the class of systems which are observable in Kalman's sense. The problem of ideal observability arises when the observer is outside of the system (1). In differential games of pursuit, one of the players is such an observer in regards to the other player. It will be noted that in problem of ideal observability the observer knows too little, that is why the class of such systems is naturally rather narrow. 
This problem and its generalizations were considered by the author (see Ref.[I]-[3]).

Now we shall go to the general problem of ideal observability. The general problem of ideal observability

It is assumed that the observer knows the signal:

$$
y(t)=C(t) z+\int_{0}^{t} D(t-s) u(s) d s, 0 \leqslant t \leqslant \Delta,
$$

where $C(t)$ is the $m \times n$-anaiytical entire matrix function,

$D(t)$ is the $m \times p$-analytical entire matrix function, $x$ is an arbitrary constant vector from $R^{n}, u \in R^{p}, u(t)$ is an arbitrary measurable function which is sumable in Lebegue's sense.

It is assumed that the observer knows $C(t), D(t)$ but does not know $u(\cdot)$. The aim of the observer is to find $Z$.

Remark 1. The presence of convolution in (1) is essentially for the obtaining of effective criterion of ideal observability.

Remark 2. In case (1), (2) $t$

$$
y(t)=G e^{t A} x_{0}+\int_{0}^{t} G e^{(t-s) A} B u(s) d s .
$$

Definition. The system (3) is ideal observable iff for every possible function $y(\cdot)$ vector $z$ is defined in a unique manner.

The necessary (but in general not sufficient) condition for the ideal observability of system (3) consists in fulfilment of the con$\operatorname{dition} \operatorname{det} \int_{0}^{\pi} C^{*}(s) C(s) d s>0$.

Theorem 1. The system (3) is ideal observable iff the function $y(t) \equiv$ $\equiv 0,0 \leqslant t \leqslant \Delta$, is generated only by $z=0$.

Remark 3. This theorem has a simple form but it is rather difficult to use it disectiy in practice.

Let us consider the function $y(t) \equiv 0$ and a couple $(x, \tilde{u}(\cdot))$ which generates it. It is possible to prove that the vector $x$ and an analytical function $u(\cdot)$ generate $y(t) \equiv 0$ also. 
Differentiating $y(t) \equiv 0$ consistently and substituting $t=0$, we have

$$
\begin{array}{ll}
C(0) z & =0 \\
C^{(1)}(0) z+D(0) u(0) & =0 \\
C^{(2)}(0) z+D^{(1)}(0) u(0)+D(0) u^{(1)}(0) & =0
\end{array}
$$

Let us put

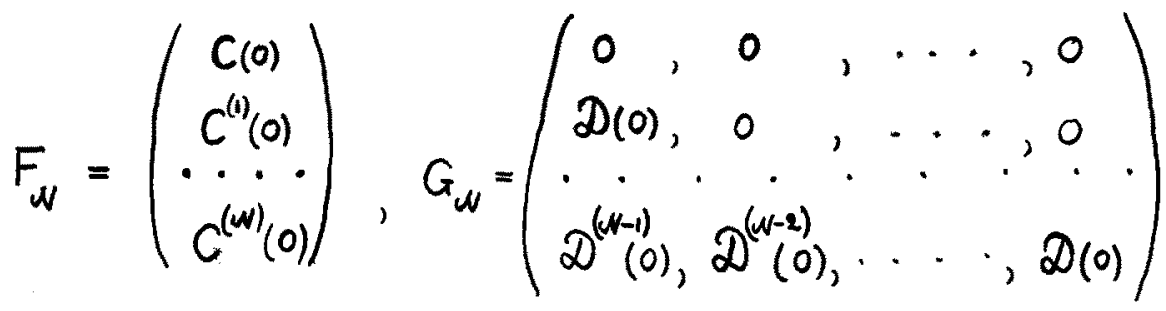

$$
\begin{aligned}
& N=1,2, \cdots .
\end{aligned}
$$

Theorem 2 (main theorem). The system (3) is ideal observable iff there exists $N \geqslant 1$ such that rank $F_{N}=n(n=\operatorname{dim} z)$ and $\operatorname{rank}\left(F_{\mathcal{N}}, G_{\mathcal{N}}\right)=\operatorname{rank} F_{\mathcal{N}}+\operatorname{rank} G_{W}$.

Lemma. One of the following conditions is valid; either exists $N_{0} \geqslant 1$ such that the conditions of theorem 2 are fulfilled for all $\mathcal{N} \geqslant N_{0}$, or one of the conditions of theorem 2 is not fulfilled for any $N \geqslant 1$.

Theorem 3. In the case of system (4) and $n \geqslant 2$, it is sufficient to verify the conditions of theorem. 2 only for

$$
\mathcal{N}_{0}=(m+1)(n-2)+1
$$

Remark 4. The Operational Calculus of Hikusinskij and the theorem of R.V.Gamkrelidze, G.I. Tharatishvili about canonical factorization of entire operational matrix function (see Ref. [4]) are used for the 
proof of theorem 2 .

Example 1. The system $\dot{x}_{1}=x_{2}, \dot{x}_{2}=u, y(t)=x_{1}(t)$ : is ideal observable.

Example 2. The system $\dot{x}_{1}=x_{2}+u_{1}, \dot{x}_{2}=u_{2}, y(t)=x_{1}(t)$

is not ideal observable.

Example 3. $y(t)=C(t) z+\int_{0}^{t} D(t-s) u(s) d s$, where $y \in R^{m}, x \in R^{n}, C(t) \neq 0, D(t) \neq 0$;

$$
C(t)=\sum_{i=k}^{\infty} C_{i} \frac{t^{i}}{i}, \quad k \geqslant 0 ; D(t)=\sum_{i=l}^{\infty} D_{i} \frac{t_{i}^{i}}{i}, l \geqslant 0 .
$$

Let rank $D_{e}=m$. It is possible to prove that under our assumptions the necessary and sufficient conditions for ideal observability of (5) are the following:

a) $k \leqslant \ell$; b) rank $\left(C_{k}^{*}, \cdots, C_{l}^{*}\right)=n$.

\section{References}

1 M.S.Nikol'skiĭ, Dokl. Akad.Nauk SSSR, v.191, N6,1970.

2 M.S.Nikol'skiI, J.Differencial'nye Uravnenija, V.XII,N4, 1971

3 M.S.Nikol'ski. I, Trudy Vicheslitelnogo Centra Akad.Nauk SSSR, collection "Issledovanie Operacil", issue N 3,1972.

4 R.V.Gamkrelidze, G.I.Kharatishvili, SIAM J.Control,v.12,N 2, 1974. 